EPJ Web of Conferences 88, 00017 (2015)

DOI: $10.1051 /$ epjconf/ 20158800017

(C) Owned by the authors, published by EDP Sciences - SIF, 2015

\title{
Symmetry energy systematics and its high density behavior
}

\author{
LiE-Wen Chen*
}

Department of Physics and Astronomy and Shanghai Key Laboratory for Particle Physics and Cosmology, Shanghai Jiao Tong University, Shanghai 200240, China

\begin{abstract}
We explore the systematics of the density dependence of nuclear matter symmetry energy in the ambit of microscopic calculations with various energy density functionals, and find that the symmetry energy from subsaturation density to supra-saturation density can be well determined by three characteristic parameters of the symmetry energy at saturation density $\rho_{0}$, i.e., the magnitude $E_{\text {sym }}\left(\rho_{0}\right)$, the density slope $L$ and the density curvature $K_{\text {sym }}$. This finding opens a new window to constrain the supra-saturation density behavior of the symmetry energy from its (sub-)saturation density behavior. In particular, we obtain $L=46.7 \pm 12.8 \mathrm{MeV}$ and $K_{\text {sym }}=-166.9 \pm 168.3$ $\mathrm{MeV}$ as well as $E_{\text {sym }}\left(2 \rho_{0}\right) \approx 40.2 \pm 12.8 \mathrm{MeV}$ and $L\left(2 \rho_{0}\right) \approx 8.9 \pm 108.7$ $\mathrm{MeV}$ based on the present knowledge of $E_{\text {sym }}\left(\rho_{0}\right)=32.5 \pm 0.5 \mathrm{MeV}$, $E_{\text {sym }}\left(\rho_{c}\right)=26.65 \pm 0.2 \mathrm{MeV}$ and $L\left(\rho_{c}\right)=46.0 \pm 4.5 \mathrm{MeV}$ at $\rho_{\mathrm{c}}=0.11$ $\mathrm{fm}^{-3}$ extracted from nuclear mass and the neutron skin thickness of Sn isotopes. Our results indicate that the symmetry energy cannot be stiffer than a linear density dependence. In addition, we also discuss the quark matter symmetry energy since the deconfined quarks could be the right degree of freedom in dense matter at high baryon densities.
\end{abstract}

*lwchen@sjtu.edu.cn

This is an Open Access article distributed under the terms of the Creative Commons Attribution License 4.0, which permits unrestricted use, distribution, and reproduction in any medium, provided the original work is properly cited 


\section{Introduction}

The nuclear matter symmetry energy, which essentially characterizes the isospin dependent part of the equation of state (EOS) of asymmetric nuclear matter, is important for understanding many questions in nuclear physics and astrophysics, including the nuclear effective interactions in asymmetric nuclear matter, the structure and stability of exotic nuclei, the reaction dynamics induced by rare isotopes, the nature and evolution of neutron stars, and the mechanism of supernova explosion [1-7]. The symmetry energy also plays an important role in some interesting issues of new physics beyond the standard model [8-12]. During the last decade, a lot of experimental, observational and theoretical efforts have been devoted to constraining the density dependence of the symmetry energy [13-18]. While significant progress has been made in determining the density behavior of the symmetry energy around saturation density $\rho_{0}\left(\sim 0.16 \mathrm{fm}^{-3}\right)$, its supra-saturation density behavior is still poorly known and remains the most uncertain property of isospin asymmetric nuclear matter. Theoretically, many experimental and observational probes have been proposed to extract information on the supra-saturation density behavior of the symmetry energy [13]. In terrestrial laboratories, heavy-ion collisions provide the only way to explore the supra-saturation density behavior of the symmetry energy under controlled conditions. To the best of our knowledge, the constraints on the suprasaturation density behavior of the symmetry energy obtained so far are all from the transport model analyses on the data of $\pi^{-} / \pi^{+}$ratio [19-25] and $n / p$ elliptic flows $[26,27]$ in heavy-ion collisions but unfortunately they are contradictive with each other, leaving a confusing situation for the community.

Conventionally, the nuclear matter EOS is defined as the binding energy per nucleon as a function of the density and a number of bulk characteristic parameters defined at saturation density $\rho_{0}$ are usually introduced to quantitatively characterize the energy of symmetric nuclear matter and the symmetry energy $[28,29]$. For example, the energy $E_{0}\left(\rho_{0}\right)$ and incompressibility $K_{0}$ of symmetric nuclear matter are the two lowest order bulk parameters for the EOS of symmetric nuclear matter while the symmetry energy magnitude $E_{\text {sym }}\left(\rho_{0}\right)$ and its slope parameter $L$ are the two lowest order bulk parameters of the symmetry energy. While several lower order bulk characteristic parameters of asymmetric nuclear matter, such as $E_{0}\left(\rho_{0}\right)$, $K_{0}, E_{\text {sym }}\left(\rho_{0}\right)$ and $L$ have been relatively well constrained or in significant progress $[13-18,30]$, yet the higher order bulk characteristic parameters are still poorly known. Actually, there has little experimental information on the 
third-order derivative parameter $J_{0}$ of symmetric nuclear matter at $\rho_{0}$ [31] and the symmetry energy curvature parameter $K_{\text {sym }}$ [29]. However, the higher order bulk characteristic parameters have been shown to be closely related to some important issues in nuclear physics and astrophysics, such as the determination of the isobaric incompressibility of asymmetric nuclear matter $[28,32]$ and the core-crust transition density and pressure in neutron stars [33-35]. In particular, within the Skyrme energy density functional, it has been proposed [29] that the higher-order curvature parameter $K_{\text {sym }}$ may play an important role in the determination of the supra-saturation density behaviors of the symmetry energy.

So far (very likely also in future), essentially all the obtained constraints on $E_{\mathrm{Sym}}(\rho)$ are based on some energy density functionals or phenomenological parameterizations of $E_{\mathrm{Sym}}(\rho)$. Therefore, it would be very interesting to see whether there exist some universal laws (systematics) for the density dependence of the symmetry energy within these functionals or parameterizations and whether one can get some useful information on the high density symmetry energy from the relatively well-known knowledge of the symmetry energy around saturation density. For all the energy density functionals or phenomenological parameterizations, the $E_{\text {sym }}(\rho)$ increases from $\rho=0$ up to a certain density around $\rho_{0}$ and then either continuously increases or decreases depending on the parameters of the energy density functionals or phenomenological parameterizations. While the parameters $E_{\mathrm{sym}}\left(\rho_{0}\right), L$ and $K_{\text {sym }}$ accurately characterize the symmetry energy density behaviors around $\rho_{0}$, their relation to the density behaviors at sub- and supra-saturation densities in various energy density functionals or phenomenological parameterizations of $E_{\text {Sym }}(\rho)$ are still unclear.

In the present talk, we report the preliminary results of the study on the systematics of the density dependence of nuclear matter symmetry energy in the ambit of microscopic calculations with various energy density functionals. We systematically analyze the relation between the parameters $E_{\text {sym }}\left(\rho_{0}\right), L$ and $K_{\text {sym }}$ defined at saturation density $\rho_{0}$ and the symmetry energy density behaviors at sub- and supra-saturation densities in various energy density functionals. In addition, since the dense matter at high baryon densities could be quark matter, we also discuss briefly the quark matter symmetry energy. 


\section{Symmetry energy systematics}

The EOS of isospin asymmetric nuclear matter, given by its binding energy per nucleon, can be expanded to 2nd-order in isospin asymmetry $\delta$ as

$$
E(\rho, \delta)=E_{0}(\rho)+E_{\mathrm{sym}}(\rho) \delta^{2}+O\left(\delta^{4}\right),
$$

where $\rho=\rho_{n}+\rho_{p}$ is the baryon density with $\rho_{n}$ and $\rho_{p}$ denoting the neutron and proton densities, respectively; $\delta=\left(\rho_{n}-\rho_{p}\right) / \rho$ is the isospin asymmetry; $E_{0}(\rho)=E(\rho, \delta=0)$ is the binding energy per nucleon in symmetric nuclear matter, and the nuclear symmetry energy is expressed as

$$
E_{\mathrm{sym}}(\rho)=\left.\frac{1}{2 !} \frac{\partial^{2} E(\rho, \delta)}{\partial \delta^{2}}\right|_{\delta=0} .
$$

Around a reference density $\rho_{r}$, the $E_{\mathrm{sym}}(\rho)$ can be expanded in $\chi_{r}=(\rho-$ $\left.\rho_{r}\right) / \rho_{r}$ as

$$
E_{\mathrm{sym}}(\rho)=E_{\mathrm{sym}}\left(\rho_{r}\right)+\frac{L\left(\rho_{r}\right)}{3} \chi_{r}+\frac{K_{\mathrm{sym}}\left(\rho_{r}\right)}{2 !} \chi_{r}^{2}+O\left(\chi_{r}^{3}\right),
$$

where $L\left(\rho_{r}\right)=\left.3 \rho_{r} \frac{\partial E_{\mathrm{sym}}(\rho)}{\partial \rho}\right|_{\rho=\rho_{r}}$ and $K_{\mathrm{sym}}\left(\rho_{r}\right)=\left.9 \rho_{r}^{2} \frac{d^{2} E_{\mathrm{sym}}(\rho)}{d \rho^{2}}\right|_{\rho=\rho_{r}}$ are, respectively, the slope and curvature parameters of the symmetry energy at $\rho_{r}$, and they are the lowest-order two bulk parameters characterizing the density behaviors of the symmetry energy around $\rho_{r}$. In particular, when the reference density $\rho_{r}$ is taken as the saturation density $\rho_{0}$, the $L\left(\rho_{r}\right)$ and $K_{\mathrm{sym}}\left(\rho_{r}\right)$ are then reduced to the famous symmetry energy slope parameter $\left.L \equiv 3 \rho_{0} \frac{d E_{\mathrm{sym}}(\rho)}{d \rho}\right|_{\rho=\rho_{0}}$ and symmetry energy curvature parameter $\left.K_{\text {sym }} \equiv 9 \rho_{0}^{2} \frac{d^{2} E_{\mathrm{sym}}(\rho)}{d \rho^{2}}\right|_{\rho=\rho_{0}}$, respectively.

To examine the symmetry energy systematics, we select a comprehensive large sample of 60 well-calibrated interactions in various energy density functionals, namely, 33 Skyrme interactions (v090, MSk7, BSk8, SKP, SKT6, SKX, BSk17, SGII, SKM*, SLy4, SLy5, MSkA, MSL0, SIV, SkSM*, kMP, SKa, Rsigma, Gsigma, SKT4, SV, SkI2, SkI5, BSK18, BSK19, BSK20, BSK21, MSL1, SAMi, SV-min, UNEDF0, UNEDF1, TOV-min), 2 Gogny interactions (D1S and D1N), 18 nonlinear RMF interactions (FSUGold, PK1s24, NL3s25, G2, TM1, NL-SV2, NL-SH, NL-RA1, PK1, NL3, NL3*, G1, NL2, NL1, IU-FSU, BSP, IUFSU*, TM1*), 2 density-dependent RMF interactions (DD-ME1 and DD-ME2), 3 point-coupling RMF interactions (DD-PC1, PC-PK1, PC-F1), and 2 relativistic HF interactions (PKO3 and PKA1). These interactions include the 46 interactions used in Ref. [36] (except BCP which is designed for density up to only $0.24 \mathrm{fm}^{-3}$ ) and other 


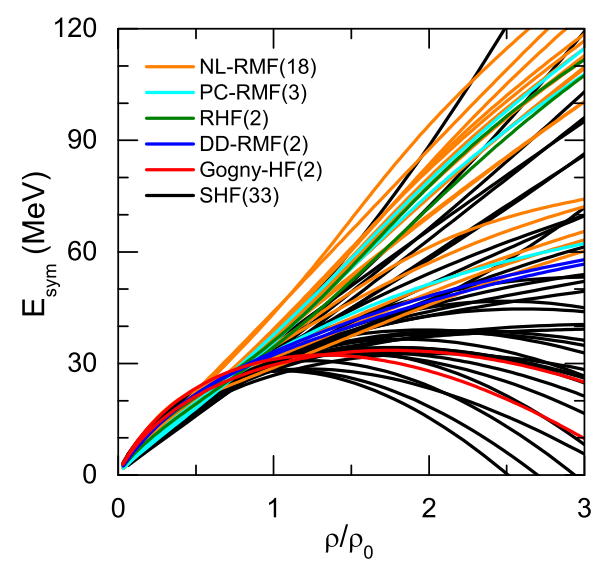

Figure 1: (Color online) The symmetry energy as a function of the density normalized by the corresponding saturation $\rho_{0}$ in various energy density functionals with 60 interactions. See text for the details.

14 interactions (i.e., BSK18, BSK19, BSK20, BSK21, MSL1, SAMi, SVmin, UNEDF0, UNEDF1, TOV-min, IU-FSU, BSP, IUFSU*, TM1*) constructed more recently. Shown in Fig. 1 is the symmetry energy as a function of the density normalized by the corresponding saturation $\rho_{0}$ with the 60 interactions. It is clearly seen that various energy density functionals predict very different density behaviors of the symmetry energy, especially at supra-saturation densities. For example, the magnitude of the symmetry energy at $2 \rho_{0}$ can be varied from about $15 \mathrm{MeV}$ to $100 \mathrm{MeV}$, depending on the models and interaction parameters. Furthermore, it is seen that some non-relativistic interactions predict negative symmetry energy at baryon densities above about $2.5 \rho_{0}$.

Around the saturation density $\rho_{0}$, the magnitude $E_{\text {sym }}(\rho)$ and the density slope $L(\rho)$ of the symmetry energy can be well approximated, respectively, by

$$
E_{\mathrm{sym}}^{*}(\rho) \equiv E_{\mathrm{sym}}\left(\rho_{0}\right)+L \chi+\frac{K_{\mathrm{sym}}}{2 !} \chi^{2},
$$

and

$$
L^{*}(\rho) \equiv L \rho / \rho_{0}+K_{\text {sym }} \chi \rho / \rho_{0},
$$

with $\chi=\left(\rho-\rho_{0}\right) / \rho_{0}$. Based on a correlation analysis within SHF approach, it has been shown [29] that $E_{\mathrm{sym}}^{*}(\rho)$ can well describe the magnitude of the symmetry energy up to $2 \rho_{0}$. How well can Eq. (4) and Eq. (5) approximate the corresponding values at densities deviated from $\rho_{0}$ in various energy density functionals? Shown in Fig. 2 is $E_{\mathrm{sym}}(\rho)$ vs $E_{\mathrm{sym}}^{*}(\rho)$ with the 60 

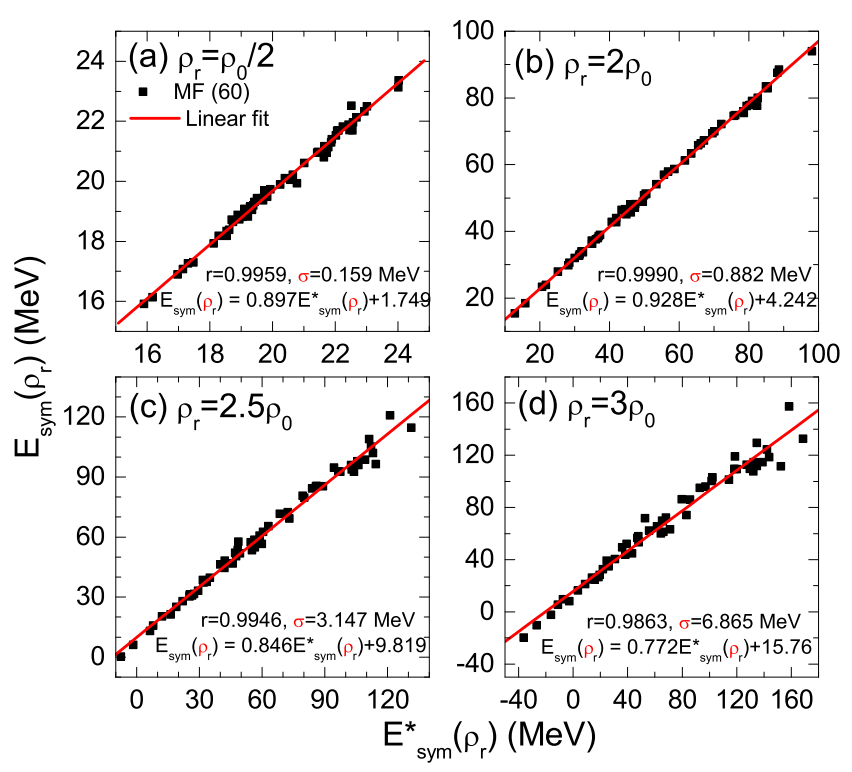

Figure 2: (Color online) $E_{\text {sym }}(\rho)$ vs $E_{\text {sym }}^{*}(\rho)$ in various energy density functionals with 60 interactions at $\rho=0.5 \rho_{0}, \rho=2 \rho_{0}, \rho=2.5 \rho_{0}$ and $\rho=3 \rho_{0}$.

interactions at $\rho=0.5 \rho_{0}, \rho=2 \rho_{0}, \rho=2.5 \rho_{0}$ and $\rho=3 \rho_{0}$. A very strong linear correlation (the Pearson linear correlation coefficient $r$ is larger than 0.98 for all the cases) is observed between $E_{\text {sym }}(\rho)$ and $E_{\text {sym }}^{*}(\rho)$ for the 60 interactions at all the four densities considered here. Similarly, Fig. 3 shows the correlation between $L(\rho)$ and $L^{*}(\rho)$ with the 60 interactions at $\rho=0.5 \rho_{0}, \rho=2 \rho_{0}, \rho=2.5 \rho_{0}$ and $\rho=3 \rho_{0}$, and again a strong linear correlation is observed between $L(\rho)$ and $L^{*}(\rho)(r$ is larger than 0.93 for the densities considered here).

The strong linear correlation between $E_{\text {sym }}(\rho)$ and $E_{\text {sym }}^{*}(\rho)$ as well as between $L(\rho)$ and $L^{*}(\rho)$ for the 60 interactions at different densities shown in Fig. 2 and Fig. 3 leads to the following relations

$$
\begin{aligned}
E_{\mathrm{sym}}(\rho) & \approx A(\rho)+B(\rho) E_{\mathrm{sym}}^{*}(\rho), \\
L(\rho) & \approx A_{L}(\rho)+B_{L}(\rho) L^{*}(\rho) .
\end{aligned}
$$

The values of the coefficients $A(\rho), B(\rho), A_{L}(\rho)$ and $B_{L}(\rho)$ generally depend the density. In particular, one can see from Fig. 2 and Fig. 3 that $A(\rho)\left(A_{L}(\rho)\right)$ is generally nonzero and $B(\rho)\left(B_{L}(\rho)\right)$ usually deviates from unit, reflecting the higher-order effects beyond the expansion in Eq. (4) and Eq. (5). We note $A(\rho) \approx 0\left(A_{L}(\rho) \approx 0\right)$ and $B(\rho) \approx 1\left(B_{L}(\rho) \approx 1\right)$ for 


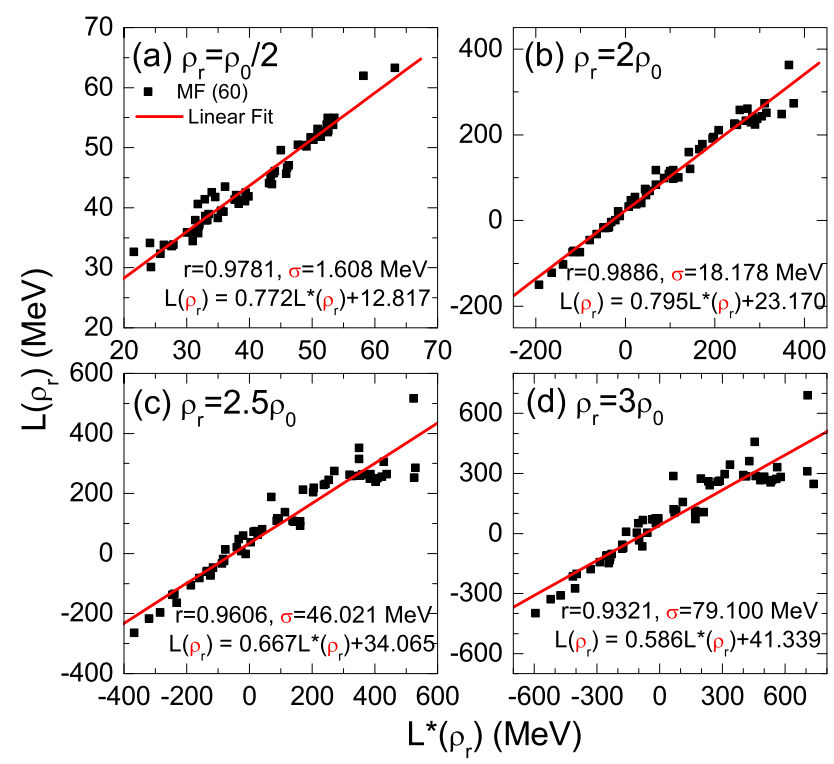

Figure 3: (Color online) Same as Fig. 2 but for $L(\rho)$ vs $L^{*}(\rho)$.

$\rho \approx \rho_{0}$ as expected. Eq. (6) and Eq. (7) establish the systematics of $E_{\text {sym }}(\rho)$ and $L(\rho)$ in terms of three characteristic parameters $E_{\text {sym }}\left(\rho_{0}\right), L$ and $K_{\text {sym }}$. It should be noted that in principe Eq. (7) can also be deduced from Eq. (6) according to the definition. We would like to point out the Eq. (6) for the systematics of $E_{\text {sym }}(\rho)$ can be safely applied in the density region from $\rho_{0} / 5$ to $3 \rho_{0}$ where the Pearson linear correlation coefficient $r$ is always larger than 0.96. Similarly, the Eq. (7) for the systematics of $L(\rho)$ can be safely applied in the density region from $\rho_{0} / 2$ to $2.5 \rho_{0}$ where the Pearson linear correlation coefficient $r$ is always larger than 0.96 .

\section{Supra-saturation density behaviors of the sym- metry energy}

The systematics of $E_{\text {sym }}(\rho)$ and $L(\rho)$ in Eq. (6) and Eq. (7) imply that the three characteristic parameters $E_{\text {sym }}\left(\rho_{0}\right), L$ and $K_{\text {sym }}$ (and thus $E_{\text {sym }}(\rho)$ and $L(\rho)$ ) can be determined once three values of either $E_{\text {sym }}(\rho)$ or $L(\rho)$ are known. This means that one can extract information on the high density behaviors of the symmetry energy from the relatively well constrained (sub)saturation density behaviors of the symmetry energy.

In recent years, several accurate constraints on the symmetry energy at 


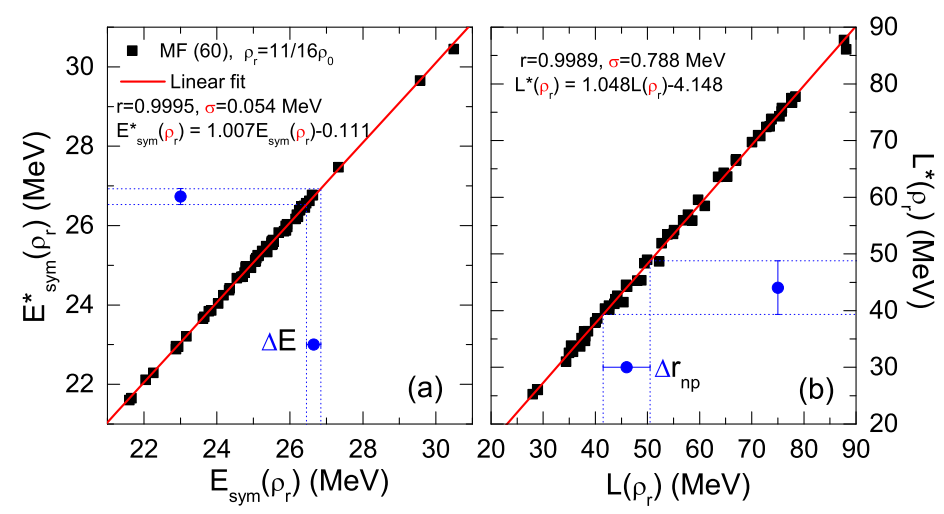

Figure 4: (Color online) $E_{\mathrm{sym}}\left(\rho_{c}\right)$ vs $E_{\mathrm{sym}}^{*}\left(\rho_{c}\right)(\mathrm{a})$ and $L\left(\rho_{c}\right)$ vs $L^{*}\left(\rho_{c}\right)$ (b) in various energy density functionals with the 60 interactions. The constraints $E_{\text {sym }}\left(\rho_{\mathrm{c}}\right)=$ $26.65 \pm 0.20 \mathrm{MeV}(\Delta E)$ and $L\left(\rho_{\mathrm{c}}\right)=46.0 \pm 4.5 \mathrm{MeV}\left(\Delta r_{n p}\right)$ obtained in Ref. [37] are also included.

subsaturation density have been obtained through analyzing nuclear structure properties of heavy nuclei. Indeed, a quite accurate constraint on the symmetry energy at the subsaturation cross density $\rho_{\mathrm{c}}=0.11 \mathrm{fm}^{-3}$, i.e., $E_{\text {sym }}\left(\rho_{\mathrm{c}}\right)=26.65 \pm 0.20 \mathrm{MeV}$, has been recently obtained from analyzing the binding energy difference of heavy isotope pairs [37]. At the same time, an accurate constraint on the density slope at $\rho_{\mathrm{c}}$, i.e, $L\left(\rho_{\mathrm{c}}\right)=46.0 \pm 4.5 \mathrm{MeV}$ has been obtained from analyzing the neutron skin data of Sn isotopes [37]. At density $\rho_{\mathrm{c}}=0.11 \mathrm{fm}^{-3}$, the systematics of $E_{\mathrm{sym}}(\rho)$ and $L(\rho)$ in Eq. (6) and Eq. (7) lead to the following expressions

$$
\begin{aligned}
E_{\mathrm{sym}}^{*}\left(\rho_{c}\right) & \approx a\left(\rho_{c}\right)+b\left(\rho_{c}\right) E_{\mathrm{sym}}\left(\rho_{\mathrm{c}}\right) \\
L^{*}\left(\rho_{c}\right) & \approx a_{L}\left(\rho_{c}\right)+b_{L}\left(\rho_{c}\right) L\left(\rho_{c}\right) .
\end{aligned}
$$

The values of coefficients $a\left(\rho_{c}\right)$ and $b\left(\rho_{c}\right)\left(a_{L}\left(\rho_{c}\right)\right.$ and $\left.b_{L}\left(\rho_{c}\right)\right)$ can be obtained from linear fitting to the correlation between $E_{\mathrm{sym}}^{*}\left(\rho_{c}\right)$ and $E_{\mathrm{sym}}\left(\rho_{\mathrm{c}}\right)\left(L^{*}\left(\rho_{c}\right)\right.$ and $\left.L\left(\rho_{c}\right)\right)$. Shown in Fig. 4 are $E_{\mathrm{sym}}\left(\rho_{c}\right)$ vs $E_{\mathrm{sym}}^{*}\left(\rho_{c}\right)$ and $L\left(\rho_{c}\right)$ vs $L^{*}\left(\rho_{c}\right)$ with the 60 interactions, and one can observe a very strong linear correlation (the Pearson linear correlation coefficient $r$ is about 0.999 for both cases) between $E_{\mathrm{sym}}^{*}\left(\rho_{c}\right)$ and $E_{\mathrm{sym}}\left(\rho_{\mathrm{c}}\right)$ as well as between $L^{*}\left(\rho_{c}\right)$ and $L\left(\rho_{c}\right)$, and these linear correlations lead to $a\left(\rho_{c}\right)=-0.111 \pm 0.111 \mathrm{MeV}, b\left(\rho_{c}\right)=1.007 \pm$ $0.004, a_{L}\left(\rho_{c}\right)=-4.148 \pm 0.358 \mathrm{MeV}$ and $b_{L}\left(\rho_{c}\right)=1.048 \pm 0.006$.

Besides $E_{\mathrm{sym}}\left(\rho_{\mathrm{c}}\right)$ and $L\left(\rho_{\mathrm{c}}\right)$, one needs another constraint condition to determine $E_{\text {sym }}\left(\rho_{0}\right), L$ and $K_{\text {sym }}$ and thus the supra-saturation density behaviors of the symmetry energy. In the present work, we further use the 


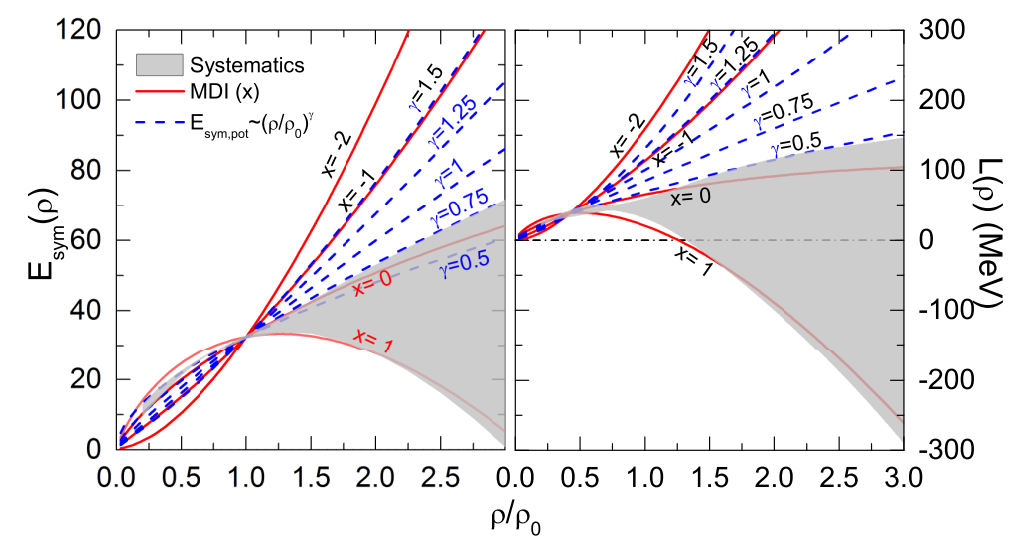

Figure 5: (Color online) Density dependence of $E_{\text {sym }}(\rho)$ and $L(\rho)$ according to the systematics in Eq. (6) and Eq. (7) with $E_{\text {sym }}\left(\rho_{0}\right)=32.5 \pm 0.5 \mathrm{MeV}, L=46.7 \pm 12.8$ $\mathrm{MeV}$ and $K_{\text {sym }}=-166.9 \pm 168.3 \mathrm{MeV}$. The results from the MDI interaction and the phenomenological parameterizations of $E_{\text {sym,pot }}(\rho) \sim\left(\rho / \rho_{0}\right)^{\gamma}$ are also include for comparison.

constraint of $E_{\mathrm{sym}}\left(\rho_{0}\right)=32.5 \pm 0.5 \mathrm{MeV}$ obtained recently from a new and more accurate finite-range droplet model analysis of the nuclear mass of the 2003 Atomic Mass Evaluation [38]. Therefore, from $E_{\mathrm{sym}}\left(\rho_{\mathrm{c}}\right)=26.65 \pm 0.20$ $\mathrm{MeV}, L\left(\rho_{\mathrm{c}}\right)=46.0 \pm 4.5 \mathrm{MeV}$ and $E_{\text {sym }}\left(\rho_{0}\right)=32.5 \pm 0.5 \mathrm{MeV}$, one can obtain $L=46.7 \pm 12.8 \mathrm{MeV}$ and $K_{\text {sym }}=-166.9 \pm 168.3 \mathrm{MeV}$. It is interesting to see that the obtained $L=46.7 \pm 12.8 \mathrm{MeV}$ is in very good agreement with other constraints extracted from terrestrial experiments, astrophysical observations, and theoretical calculations with controlled uncertainties [13-18]. The obtained $K_{\text {sym }}=-166.9 \pm 168.3 \mathrm{MeV}$ also agrees well with the result $K_{\text {sym }}=-100 \pm 165 \mathrm{MeV}$ [29] obtained from a correlation analysis within SHF approach.

Based on $E_{\text {sym }}\left(\rho_{0}\right)=32.5 \pm 0.5 \mathrm{MeV}, L=46.7 \pm 12.8 \mathrm{MeV}$ and $K_{\text {sym }}=$ $-166.9 \pm 168.3 \mathrm{MeV}$, one then can obtain $E_{\mathrm{sym}}(\rho)$ and $L(\rho)$ according to the systematics in Eq. (6) and Eq. (7), and the results are shown in Fig. 5. For comparison, we also include the results from the MDI interaction [39] with $x=1,0,-1$ and -2 and the phenomenological parameterizations of $E_{\text {sym,pot }}(\rho) \sim\left(\rho / \rho_{0}\right)^{\gamma}[26,40]$ for the potential energy part of the symmetry energy with $\gamma=0.5,0.75,1.0,1.25$ and 1.5 . We would like to point out that the MDI interaction and the parameterizations of $E_{\text {sym,pot }}(\rho) \sim\left(\rho / \rho_{0}\right)^{\gamma}$ have been extensively applied in transport model simulations of heavy ion collisions. One can see from Fig. 5 that the present analysis based on the symmetry energy systematics with $E_{\text {sym }}\left(\rho_{0}\right)=32.5 \pm 0.5 \mathrm{MeV}, L=46.7 \pm$ 
$12.8 \mathrm{MeV}$ and $K_{\text {sym }}=-166.9 \pm 168.3 \mathrm{MeV}$ favors a softer symmetry energy and suggests that the symmetry energy cannot be stiffer than a linear density dependence.

In particular, at the supra-saturation density of $2 \rho_{0}$, we find $E_{\mathrm{sym}}\left(2 \rho_{0}\right)=$ $40.2 \pm 12.8 \mathrm{MeV}$ and $L\left(2 \rho_{0}\right)=8.9 \pm 108.7 \mathrm{MeV}$. We note that these values are in nice agreement with the variational many-body theory calculation with WFF1 interaction [41] which can give a good description on the recent observation of heavy neutron stars with radius of $9.1_{-1.5}^{+1.3} \mathrm{~km}[42]$.

\section{Quark matter symmetry energy}

At extremely high baryon density, the main degree of freedom could be the deconfined quark matter rather than the confined baryon matter, and there the quark matter symmetry energy should be involved for the properties of isospin asymmetric quark matter (isospin symmetry is still satisfied in quark matter). The isospin asymmetric quark matter could be produced in ultrarelativistic heavy ion collisions induced by neutron-rich nuclei and it could also exist in compact stars such as neutron stars or quark stars. Although significant progress has been made in understanding the density dependence of the nuclear matter symmetry energy, there has little information on the density dependence of the quark matter symmetry energy. Theoretically, it is difficult to calculate the quark matter symmetry energy since the $a b$ initio Lattice QCD simulations does not work at finite baryon density while perturbative QCD only works at extremely high baryon density.

Similarly as in the case of nuclear matter, the EOS of quark matter consisting of $u, d$, and $s$ quarks, defined by its binding energy per baryon number, can be expanded in isospin asymmetry $\delta_{q}$ as

$$
E\left(n_{B}, \delta, n_{s}\right)=E_{0}\left(n_{B}, n_{s}\right)+E_{\mathrm{sym}}\left(n_{B}, n_{s}\right) \delta_{q}^{2}+\mathcal{O}\left(\delta_{q}^{4}\right),
$$

where $E_{0}\left(n_{B}, n_{s}\right)=E\left(n_{B}, \delta_{q}=0, n_{s}\right)$ is the binding energy per baryon number in three-flavor $u-d-s$ quark matter with equal fraction of $u$ and $d$ quarks; the quark matter symmetry energy $E_{\mathrm{sym}}\left(n_{B}, n_{s}\right)$ is expressed as

$$
E_{\mathrm{sym}}\left(n_{B}, n_{s}\right)=\left.\frac{1}{2 !} \frac{\partial^{2} E\left(n_{B}, \delta, n_{s}\right)}{\partial \delta_{q}^{2}}\right|_{\delta_{q}=0} .
$$

The isospin asymmetry of quark matter is defined as

$$
\delta_{q}=3 \frac{n_{d}-n_{u}}{n_{d}+n_{u}},
$$


which equals to $-n_{3} / n_{B}$ with the isospin density $n_{3}=n_{u}-n_{d}$ and $n_{B}=\left(n_{u}+n_{d}\right) / 3$ for two-flavor $u-d$ quark matter. We note that the above definition of $\delta_{q}$ for quark matter has been extensively used in the literature [43-47], and one has $\delta_{q}=1(-1)$ for quark matter converted by pure neutron (proton) matter according to the nucleon constituent quark structure, consistent with the conventional definition for nuclear matter, namely, $\frac{\rho_{n}-\rho_{p}}{\rho_{n}+\rho_{p}}=-n_{3} / n_{B}$. In Eq. (10), the absence of odd-order terms in $\delta_{q}$ is due to the exchange symmetry between $u$ and $d$ quarks in quark matter when one neglects the Coulomb interaction among quarks. The higher-order coefficients in $\delta_{q}$ are shown to be very small in various model calculations [47].

It has been demonstrated recently [47] that the isovector properties of quark matter may play an important role in understanding the properties of strange quark matter and quark stars. If the recently discovered heavy pulsars PSR J1614-2230 [48] and PSR J0348+0432 [49] with mass around $2 M_{\odot}$ were quark stars, they can put important constraint on the isovector properties of quark matter, especially the quark matter symmetry energy. Within the confined-isospin-density-dependent-mass (CIDDM) model [47], in particular, it has been shown that the two-flavor $u$ - $d$ quark matter symmetry energy should be at least about twice that of a free quark gas or normal quark matter within conventional NJL model in order to describe the PSR J1614-2230 and PSR J0348+0432 as quark stars.

\section{Summary}

We have explored the systematics of the density dependence of the symmetry energy in the ambit of microscopic calculations with various energy density functionals. Our results indicate that the symmetry energy magnitude $E_{\text {sym }}(\rho)$ and its density slope $L(\rho)$ from subsaturation density to suprasaturation density can be essentially determined by three parameters defined at saturation density $\rho_{0}$, i.e., the magnitude $E_{\text {sym }}\left(\rho_{0}\right)$, the density slope $L$ and the density curvature $K_{\mathrm{sym}}$. This finding implies that three values of $E_{\text {sym }}(\rho)$ or $L(\rho)$ essentially determine $E_{\text {sym }}(\rho)$ and $L(\rho)$ in large density region. In particular, using $E_{\mathrm{sym}}\left(\rho_{c}\right)=26.65 \pm 0.2 \mathrm{MeV}$ and $L\left(\rho_{c}\right)=46.0 \pm 4.5$ $\mathrm{MeV}$ at $\rho_{\mathrm{c}}=0.11 \mathrm{fm}^{-3}$ extracted from isotope binding energy difference and neutron skin of Sn isotopes together with $E_{\text {sym }}\left(\rho_{0}\right)=32.5 \pm 0.5 \mathrm{MeV}$ obtained from finite-range droplet model analysis of nuclear binding energy, we obtain $L=46.7 \pm 12.8 \mathrm{MeV}$ and $K_{\text {sym }}=-166.9 \pm 168.3 \mathrm{MeV}$ as well as $E_{\text {sym }}\left(2 \rho_{0}\right) \approx 40.2 \pm 12.8 \mathrm{MeV}$ and $L\left(2 \rho_{0}\right) \approx 8.9 \pm 108.7 \mathrm{MeV}$. These results favor a soft to roughly linear density dependence of the symmetry energy. 
We have also discussed the quark matter symmetry energy, which has been shown to play an important role in understanding the properties of strange quark matter and quark stars. In particular, it has been suggested that the two-flavor $u$ - $d$ quark matter symmetry energy should be at least about twice that of a free quark gas or normal quark matter within conventional NJL model in order to describe the recently discovered heavy pulsars PSR J1614-2230 and PSR J0348+0432 with mass around $2 M_{\odot}$ as quark stars.

The author thanks Wei-Zhou Jiang, Che Ming Ko, Bao-An Li, De-Hua Wen, Hermann Wolter, and Jun Xu for useful discussions. This work was supported in part by the Major State Basic Research Development Program (973 Program) in China under Contracts No. 2015CB856904 and No. 2013CB834405, the National Natural Science Foundation of China under Grants No. 11275125 and No. 11135011, the "Shu Guang" project supported by Shanghai Municipal Education Commission and Shanghai Education Development Foundation, the Program for Professor of Special Appointment (Eastern Scholar) at Shanghai Institutions of Higher Learning, and the Science and Technology Commission of Shanghai Municipality (11DZ2260700).

\section{References}

[1] B.A. Li, C.M. Ko, and W. Bauer, Int. Jour. Mod. Phys. E 7, 147 (1998).

[2] P. Danielewicz, R. Lacey, and W.G. Lynch, Science 298, 1592 (2002).

[3] J.M. Lattimer and M. Prakash, Science 304, 536 (2004); Phys. Rep. 442, 109 (2007).

[4] W. Steiner, M. Prakash, J.M. Lattimer, and P.J. Ellis, Phys. Rep. 411, 325 (2005).

[5] V. Baran, M.Colonna, V. Greco, and M. Di Toro, Phys. Rep. 410, 335 (2005).

[6] L.W. Chen, C.M. Ko, B.A. Li, and G.C. Yong, Front. Phys. China 2, 327 (2007).

[7] B.A. Li, L.W. Chen, and C.M. Ko, Phys. Rep. 464, 113 (2008).

[8] C.J. Horowitz, S.J. Pollock, P.A. Souder and R. Michaels, Phys. Rev. C 63, 025501 (2001). 
[9] T. Sil, M. Centelles, X. Viñas, and J. Piekarewicz, Phys. Rev. C 71, 045502 (2005).

[10] D.H. Wen, B.A. Li, and L.W. Chen, Phys. Rev. Lett. 103, 211102 (2009).

[11] H. Zheng, Z. Zhang, and L.W. Chen, J. Cosmol. Astropart. Phys. 08, 011 (2014).

[12] H. Zheng, K.J. Sun, and L.W. Chen, Astrophys. J., 2015 (in press) [arXiv:1408.2926].

[13] Topical Issue Nuclear Symmetry Energy edited by Bao-An Li, Angels Ramos, Giuseppe Verde, Isaac Vidana, Eur. Phys. J. A 50, (2014).

[14] B.M. Tsang et al., Phys. Rev. C 86, 015803 (2012).

[15] J.M. Lattimer, Ann. Rev. Nucl. Part. Sci. 62, 485 (2012).

[16] L.W. Chen, Nuclear Structure in China 2012: Proceedings of the 14th National Conference on Nuclear Structure in China (NSC2012) (World Scientific, Singapore, 2012), pp. 43-54 [arXiv:1212.0284].

[17] B.A. Li et al., J. Phys.: Conf. Series 413, 012021 (2013) [arXiv:1212.1178].

[18] C.J. Horowitz et al., J. Phys. G 41, 093001 (2014).

[19] Z. Xiao, B.A. Li, L.W. Chen, G.C. Yong, and M. Zhang, Phys. Rev. Lett. 102, 062502 (2009); M. Zhang et al., Phys. Rev. C 80, 034616 (2009).

[20] Z.Q. Feng and G.M. Jin, Phys. Lett. B683, 140 (2010).

[21] J. Xu, C.M. Ko, Y. Oh, Phys. Rev. C 81, 024910 (2010).

[22] W.J. Xie, J. Sun, L. Zhu, and F.S. Zhang, Phys. Lett. B718, 1510 (2013).

[23] J. Xu, L.W. Chen, C.M. Ko, B.A. Li, and Y.G. Ma, Phys. Rev. C 87, 067601 (2013).

[24] J. Hong and P. Danielewicz, Phys. Rev. C 90, 024605 (2014).

[25] T. Song and C.M. Ko, arXiv:1403.7363. 
[26] P. Russotto et al., Phys. Lett. B697, 471 (2011).

[27] M.D. Cozma, Y. Leifels, W. Trautmann, Q. Li, and P. Russotto, Phys. Rev. C 88, 044912 (2013).

[28] L.W. Chen et al., Phys. Rev. C 80, 014322 (2009).

[29] L.W. Chen, Sci. China: Phys. Mech. Astro. 54 (Suppl. 1), s124 (2011).

[30] D.H. Youngblood, H.L. Clark, and Y.-W. Lui, Phys. Rev. Lett. 82, 691 (1999).

[31] B.J. Cai and L.W. Chen, arXiv:1402.4242.

[32] L.W. Chen, Sci. China Ser. G 52, 1494 (2009).

[33] J. Xu, L.W. Chen, B.A. Li, and H.R. Ma, Phys. Rev. C 79, 035802 (2009).

[34] J. Xu, L.W. Chen, B.A. Li, and H.R. Ma, Astrophys. J. 697, 1549 (2009).

[35] C. Ducoin, J. Margueron, and C. Providência, Europhys. Lett. 91, 32001 (2010).

[36] X. Roca-Maza, M. Centelles, X. Viñas, and M. Warda, Phys. Rev. Lett. 106, 252501 (2011).

[37] Z. Zhang and L.W. Chen, Phys. Lett. B726, 234 (2013).

[38] P. Moller, W.D. Myers, H. Sagawa, and S. Yoshida, Phys. Rev. Lett. 108, 052501 (2012).

[39] L.W. Chen, C.M. Ko, and B.A. Li, Phys. Rev. Lett. 94, 032701 (2005).

[40] M.B. Tsang et al., Phys. Rev. Lett. 102, 122701 (2009).

[41] R.B. Wiringa, V. Fiks, and A. Fabrocini, Phys. Rev. C 38, 1010 (1988).

[42] S. Guillot, M. Servillat, N.A. Webb, and R.E. Rutledge, Astrophys. J. 772, 7 (2013).

[43] M. Di Toro, A. Drago, T. Gaitanos, V. Greco, and A. Lavagno, Nucl. Phys. A775, 102 (2006)

[44] G. Pagliara and J. Schaffner-Bielich, Phys. Rev. D 81, 094024 (2010). 
[45] M. Di Toro, V. Baran, M. Colonna, and V. Greco, J. Phys. G 37, 083101(2010).

[46] G.Y. Shao, M. Colonna, M. Di Toro, B. Liu, and F. Matera, Phys. Rev. D 85, 114017 (2012).

[47] P.C. Chu and L.W. Chen, Astrophys. J. 780, 135 (2014).

[48] P. Demorest, T. Pennucci, S. Ransom, M. Roberts, and J. Hessels, Nature 467, 1081 (2010).

[49] J. Antoniadis et al., Science 340, 6131 (2013). 